Share Your Innovations through JACS Directory

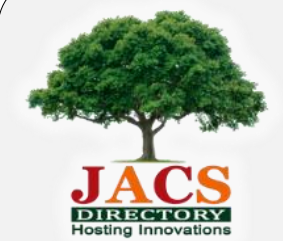

Journal of Environmental Science and Pollution Research

Visit Journal at http://www.jacsdirectory.com/jespr

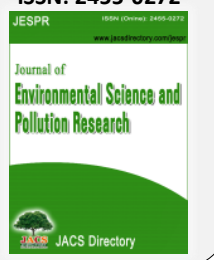

\title{
Mechanical, Chemical, Structural, and Radiological Changes in Pigeon Bone, Associated with The Dietary Intake of Nickel Recovery Slag
}

\author{
E. Galiano*, M. Lapointe \\ Department of Physics, Laurentian University, 935 Ramsey Lake Rd., Sudbury Ontario, P3E 2C6, Canada.
}

\section{AR T I C LE D E T A I L S}

\section{Article history:}

Received 22 June 2019

Accepted 05 July 2019

Available online 15 July 2019

\section{Keywords:}

Bone Metabolism

Iron Overload

Bone Mineral Density

Young's Modulus

Breaking Strength

\begin{abstract}
A B S T R A C T
Slag from nickel smelting operations in the Sudbury basin in Ontario, Canada, has become ubiquitous. This material rich in heavy metals such as iron, upon ingestion has the potential to effect physical, radiological, chemical, mechanical, and structural changes in biological systems. In this work, we analyzed the effects of slag ingestion through diet, on several quantitative and qualitative parameters of the tibio-tarsal bones in pigeons (Columbia Livia Domestica). The specimens were maintained on a seed diet and divided into a control group $(n=9)$ provided (normal) clean limestone grit, and an experimental group $(\mathrm{n}=9)$ provided slag-based grit, both for a period of one year. Their tibio-tarsal bones were harvested for analysis. Quantitative analytical methods included conventional density measurements, caliper-based cortical thickness measurements, bone mineral density measurements using Dual Energy X-ray Absorptiometry (DEXA), calcium and iron concentration measurements using mass spectrometry, and the determination of Young's Moduli and ultimate breaking strength (both in compression) using a universal testing machine (UTM). Qualitative microscopy studies, both optical and electron - including energy dispersive spectroscopy (EDS) - were carried out for both sample groups. A Welch's t-test (single tail) was used to compare means of the seven quantitative parameters between control and experimental samples; in six of these parameters, a statistically significant difference was found ( $p \leq$ 0.05). Microscopy, both optical and electron - coupled with EDS analysis - revealed structural differences in bone between the two groups. Engineering hardness testing of grit types in the dietary components revealed that the slag ingested by the experimental group was approximately twice as hard (Brinell and Rockwell hardness) as the limestone grit in the control group diet. We conclude that slag ingestion through diet in the species examined, is associated with measurable changes in mechanical, chemical, structural, and radiological properties of the tibio-tarsal bones.
\end{abstract}

\section{Introduction}

Slag tailings containing high concentrations of base metals from over 130 years of continuous nickel smelting operations in the Sudbury, Ontario basin in Canada, have become ubiquitous. Among its engineering uses are road construction, laying of railway beds, bridge building, construction of retaining buttresses, break walls, causeways, and dikes, surface stabilization of inclines and ravines, production of cement and concrete aggregates, and occasional use as a base substrate for parking lots and airport runways [1,2]. This material rich in heavy metals particularly iron - upon ingestion has the potential to effect physical, radiological, chemical, mechanical, and structural changes in bony structures of biological systems. Micro chemical analysis has revealed that Sudbury basin nickel recovery slag contains approximately $34.7 \pm 0.6 \% \mathrm{Fe}$ by weight [1]. Most birds - and granivorous species in particular - depend on a continuous uptake of pebble-sized grit to assist in the grinding functions of the gizzard, and are known to routinely obtain such pebbles from available sources within their home range environment. In this work, we extend previous work and analyze the effects of slag ingestion through diet, on seven different quantitative, and two qualitative parameters of the tibio-tarsal bones in pigeons (Columbia Livia Domestica) [1]. The specimens were divided into a control group $(n=9)$, which were provided a (normal) grit source of pristine limestone, and an experimental group ( $\mathrm{n}$ = 9) fed slag-based grit, both for a period of 1 year. Their tibio-tarsal bones were then harvested for analysis. Quantitative analytical methods included measurement of compact bone thickness of the tibio-tarsus (TH), conventional density measurements (CD), bone mineral density (BMD) measurements using Dual Energy X-Ray Absorptiometry (DEXA), calcium and iron concentrations ( $\mathrm{Ca}$ and $\mathrm{Fe}$ respectively) using mass

*Corresponding Author:egalianoriveros@laurentian.ca(E. Galiano) spectrometry, and the determination of Young's Moduli (YM) and ultimate breaking strength (BS) (both in compression) using a universal testing machine (UTM). Qualitative microscopy studies, both optical and scanning electron including energy dispersive spectrometry (EDS), were also carried out for both treatment groups. In addition, hardness testing of limestone and slag was undertaken. This work was performed in accordance with an animal care protocol approved by the University of Guelph (where husbandry, sacrifice, and organ harvesting of the samples took place) Animal Care Committee, on behalf of the Canadian Council on Animal Care.

\section{Experimental Methods}

The specimens were sacrificed and frozen after one year of controlled feeding. Selection, feeding, and husbandry of the specimens have been previously described [1]. Tibio-tarsal bones were excised from the frozen birds. Any remaining soft tissue surrounding the bone as well as the attached fibula, were removed. To maintain consistency, only right tibiotarsi were utilized for destructive mass spectrometry measurements. All other experimental procedures thus utilized left tibio-tarsi. All samples were properly labeled and re-frozen for preservation. Upon re-thawing, a Dremel@ moto tool (Dremel Model 4300-9/64 High Performance Variable Speed Rotary Tool, Dremel Inc. Racine, WI, USA) was used to remove the two epiphyses from the diaphysis. Marrow was removed with a sewing pin followed by a pipe cleaner, resulting in a smooth hollow cylinder, followed by oven drying at $65^{\circ} \mathrm{C}$ for 48 hours, as suggested by Kim et al. [3].

A caliper (Mitsuyo model 500-196-30, Mitsuyo America Corporation, Aurora IL) was used to measure the outer and inner diameters of six randomly selected bones from each group ( $\mathrm{n}=6$ each, $\mathrm{n}=12$ total). In each case, the average of the three measurements was used for computational purposes. Compact bone thickness ( $\mathrm{TH}$ ) for each sample was calculated by taking the difference between the mean inner and outer diameters. 
Conventional (or ordinary) density (CD) measurements of all 18 samples were made $(\mathrm{n}=9$ each group, $\mathrm{n}=18$ total). A sealed scale was used to minimize airflow interference (Escali Model L-125, Escali Corp., Burnville, MN, USA) to determine the mass of each sample. Sample volumes were obtained by careful measurement of water displacement upon full sample immersion in a $25 \mathrm{~mL}$ pyrex flask. The volumes of water before and after sample immersions were compared, with sample volume taken as the difference between pre- and post-immersion water volumes. Sample immersion times sufficient to allow for complete sample saturation and degassing were observed.

Bone mineral density (BMD) measurements involve the use of a dual energy x-ray (DEXA) beam, which is directed at the sample/patient. The energy distribution of the beam after passing through the sample/patient is analyzed to infer a ratio of high atomic number material (bone) to low atomic number material (soft tissue) [4]. The technique produces an "area density" number for the bone sample in units of $\mathrm{g} / \mathrm{cm}^{2}$, referred to clinically as the "bone mineral density" or BMD. The method is the accepted standard for the diagnosis of osteoporosis in humans. Commercially available DEXA scanners for clinical use are designed to scan human patients, and are therefore equipped with specific data acquisition protocols which are not optimized for animal studies. Therefore an "equivalent" acquisition protocol specific to our samples had to be developed for this work. In order to simulate a soft tissue component - a prerequisite for the machine software to function properly - the bone samples were surrounded with bags of white rice. With these considerations, samples ( $n=6$ each group, $n=12$ total) from the control and experimental groups were subjected to DEXA scanning (Lunar Prodigy Advance model PA 301850, GE Healthcare, Madison, WI).

Mass spectrometry is a high-precision, high-sensitivity method for elemental analysis. It is based on the physical principle of the electromagnetic separation of atomic nuclei of different masses. Right side frozen tibio-tarsi bones from all samples in both groups $(\mathrm{n}=9$ each group, $\mathrm{n}=18$ total) were thawed and fragmented into small pieces; fat inclusions were removed. Samples weighing $0.5 \pm 0.01 \mathrm{~g}$ were digested in $6.4 \mathrm{~mL}$ of $\mathrm{HNO}_{3}$ and $1.6 \mathrm{~mL}$ of $\mathrm{H}_{2} \mathrm{O}_{2}(30 \%)$ using pulsatile microwave heating (EMS820 Precision Pulsed Laboratory Microwave Oven, Hatfield, PA, USA) and brought to volume $(25.0 \pm 0.1 \mathrm{~mL})$ with deionized water. The digested samples were subjected to mass spectrometry with special emphasis on $\mathrm{Ca}$ and $\mathrm{Fe}$ measurements (Perkin-Elmer Model 7.03 flame atomic absorption spectrophotometer, Shelton, CT, USA). The mean values of Ca and $\mathrm{Fe}$ concentrations were recorded. $\mathrm{Ca}$ and $\mathrm{Fe}$ concentrations are reported in standard units of parts per million (ppm).

Young's Modulus (YM) is a physical parameter which quantifies the elasticity of a solid. It is formally defined as the ratio of the solid's stress to its strain, under a progressively applied compressive - or tensile - load. It is the physical analogue of Hooke's constant for a spring, applied to a solid [5]. Breaking strength (BS) is the minimum axial force required to induce mechanical failure of the solid, and clearly its measurement is destructive in nature. It is common practice in the engineering testing of materials, to acquire YM and BS data simultaneously, using a so-called Universal Testing Machine (UTM). This machine consists of a hydraulic piston which compresses the sample at a carefully controlled rate, recording sample compression and applied force, up to the point of failure. Based on sample geometry, stress-strain data are generated and Young's Modulus is extracted by a "best-fit of the slope" algorithm. The machine automatically produces a stress-strain curve, a best fit value of YM (in metric units of $\mathrm{MPa}$ ), and a breaking strength (BS) value (in metric units of $\mathrm{N}$ ). Left side tibio-tarsal bones of the control and experimental groups $(n=7$ and $n=8$ respectively) were subjected to destructive compressional YM and BS measurements (Tinius Olsen Model 2000 SL UTM, Horsham, PA, USA). During initial testing, three samples (one experimental, two control) were accidentally destroyed before useful data could be generated. Therefore, useful data was only generated for seven control and eight experimental samples. Note that only the compressional performance of tibio-tarsal bones is relevant, as these are never physiologically subjected to tensile loads in a natural environment. As an expected outcome of this type of testing, all samples were destroyed in the process, and therefore this was the last procedure requiring pristine samples in our investigations. Subsequent microscopy work was performed with remaining fragments of the samples.

As part of this work, additional qualitative differential analyses of samples from the two groups were undertaken; In particular, electron and optical microscopy studies, followed by energy dispersive spectroscopy (EDS). Electron micrographs of mid-diaphysis cortical tibio-tarsi of representative samples (one each) from the control and experimental groups respectively, were obtained (JEOL model 6400 Scanning Electron Microscope, equipped with Energy Dispersive Spectroscopy (EDS) capabilities, JEOL Corp., Tokyo, Japan). Image acquisition parameters were: accelerating voltage $30 \mathrm{kV}$, beam current $1.0 \times 10^{-11} \mathrm{~A}$. Successive https://doi.org/10.30799/jespr.171.19050205 trials established that no special sample preparation techniques were deemed necessary, thus no metallic coating was applied to the samples. Both samples were subjected to energy dispersive spectroscopy (EDS) during imaging. In this technique, semi-elastic electron-electron interactions between the microscope's electron beam, and orbital electrons from sample atoms, produce electron vacancies. These are promptly filled by higher energy electrons, resulting in the emission of characteristic X-rays [6]. These X-rays are detected and compared to a stored library, which after processing produces an elemental breakdown of the sample on a fractional mass basis. The rectangles in the micrographs (in Figs. 2a and b) are the sample areas interrogated for EDS analysis. Electron microscopy and EDS spectroscopy studies were followed up with conventional microscopy of the same two samples, with particular attention to the cortical, mid-diaphysis regions (Nikon Upright Optiphot Model 200 microscope, Nikon Inc. Shinagawa, Tokyo, Japan, equipped with Proscope model HR camera, Proscope Inc., Oregon City, OR, USA).

As a final task, in order to further our understanding of the mechanical differences between dietary components of the control and experimental groups - which may be associated with physiological changes - hardness testing of the dietary grit types was undertaken. A sample of pristine limestone provided to the control group, and a sample of metal-rich slag grit supplied to the experimental group, were independently tested. Each sample underwent Brinell Hardness testing (Louis Small Inc. Model 8BLP Brinell Hardness Tester, Cincinatti, OH, USA), and Rockwell Hardness testing (Service Diamond Tool Co. Model 1JS Rockwell Hardness Tester, Ferndale, MI, USA). Both the Brinell and Rockwell testing protocols are in standard use in engineering and materials sciences. There are operational differences between these protocols, but they are considered generally equivalent, and typically a thorough engineering analysis will include subjecting a sample to both protocols independently, as they are nondestructive $[7,8]$.

\section{Results and Discussion}

All quantitative results are given as a mean \pm mean standard error. The calculations were done using the statistics package in Microsoft Excel $2010 ®$. All parameters were subjected to a single tail Welch's t-test which allows for unequal sample sizes and variances - to determine statistical significance in differences in means between the two groups. A $\mathrm{p} \leq 0.05$ threshold was selected to indicate statistical significance, as indicated by ANOVA.

The mean cortical bone thickness (TH) for the experimental and control groups (both $\mathrm{n}=6$ ) were $0.48 \pm 0.03 \mathrm{~mm}$ and $0.61 \pm 0.07 \mathrm{~mm}(\mathrm{p}=0.0500)$ respectively, representing an approximate reduction in mean thickness of $21 \%$ for the experimental group. This loss suggests that slag ingestion is associated with loss of cortical bone thickness, consistent with previously reported results by Tsay et al. and De Vernejoul et al., in iron overloaded murine and porcine models $[9,10]$.

The mean conventional density (CD) for the experimental and control groups (both $\mathrm{n}=9$ ) group were $1.7698 \pm 0.2223 \mathrm{~g} / \mathrm{cm}^{3}$ and $1.9785 \pm$ $0.1948 \mathrm{gm} / \mathrm{cm}^{3}$ ( $\left.\mathrm{p}=0.0251\right)$ respectively, representing an approximate loss in mean density of $10 \%$ for the experimental group. These results are compatible with iron overload induced loss of bone mineral content reported in avian and porcine models [10-14]. To further substantiate iron overload in bone, we later present optical microscopy and spectrometric evidence of the presence of an abnormal localized iron deposit in bone in an experimental group sample. No previous reports of decreased bone density in slag fed animals were found in the literature.

The mean bone mineral density (BMD) for the experimental and control groups (both $\mathrm{n}=6$ ) were $0.143 \pm 0.005 \mathrm{~g} / \mathrm{cm}^{2}$ and $0.152 \pm 0.005 \mathrm{~g} / \mathrm{cm}^{2}(\mathrm{p}$ $=0.0089$ ) respectively, representing an approximate loss in mean density of $6 \%$ for the experimental group. Relatively elevated data signal to noise ratios of 3289 and 2624 for the control group and experimental groups respectively, resulted in a highly significant difference ( $p$ value of 0.0089 ). These results are consistent with those of Ward and collaborators, who reported a reduction in BMD associated with increased Fe levels in birds [15]. Conrad et al. and later Hester et al., reported a correlation between reduced BMD values in poultry femora, with associated osteoporotic-like conditions $[16,17]$. In humans, a reduced BMD is strongly correlated with clinical osteoporosis [18]. Furthermore, Kim and collaborators have reported that in humans, iron overload (elevated serum ferritin levels), is associated with reduced BMD levels [19].

The mean Ca concentrations measured by mass spectrometry for the experimental and control groups (both $\mathrm{n}=9$ ) were $70912.8 \pm 11952.7$ ppm and $82621.8 \pm 11046.7 \mathrm{ppm}(\mathrm{p}=0.0272)$, representing an approximate loss of $14 \%$ of calcium content for the experimental group. This loss suggests that dietary slag (i.e. iron) is associated with reduced $\mathrm{Ca}$ uptake/retention in bone. The mean Fe concentration measurements

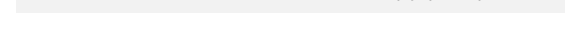


reveal a remarkable increase in the experimental group: $242.76 \pm 190.35$ ppm for the experimental group vs. $80.87 \pm 37.41 \mathrm{ppm}$ for the control group (both $\mathrm{n}=9, \mathrm{p}=0.0127$ ). This represents about a $200 \%$ increase in Fe concentration! The large error interval for the experimental group is possibly associated with the non-homogenous deposition of iron in the samples, as supported by optical microscopy results and Energy Dispersive Spectroscopy (EDS) measurements. Inverse correlations between bone calcium content and iron overload in animal models have been reported in the literature. For example, Tsay et al. reported an inverse correlation between iron overload and bone calcium content in mice [9]. Likewise, Vernejoul and collaborators described alterations in bony calcium metabolism in porcines subjected to iron overload [10]. Dorrestein et al. reported elevated Fe levels in bones in pigeons fed a high $\mathrm{Fe}$ concentration diet, but did not address any changes in $\mathrm{Ca}$ concentrations [20].

The mean breaking strength (BS) values for the control and experimental groups $(\mathrm{n}=7$ and $\mathrm{n}=8$ respectively) were $451.57 \pm 133.78$ $\mathrm{N}$ and $328.50 \pm 139.62 \mathrm{~N}(\mathrm{p}=0.0500)$ respectively, representing an approximate reduction of $27 \%$ for the experimental group. Kim and collaborators have reported BS measurements in poultry bones; however, they did not examine samples with modified diets [3]. In work that has some similarities to our own, Huyghebaert and collaborators investigated the effects of dietary fluoride in the breaking strength of the leg bones in broilers [21]. From a structural point of view, this reduction in breaking strength is consistent with our cortical thickness (TH) measurements mentioned earlier, where we report a $21 \%$ reduction in the mean thickness of the experimental group. Zhang and collaborators have reported comparable results in hen bones, in which a reduction in BS was associated with reduced bone dimensions [22].

The mean Young's Moduli (YM) values for the control and experimental groups ( $\mathrm{n}=7$ and $\mathrm{n}=8$ respectively) were $3483.6 \pm 1853.7 \mathrm{MPa}$ and $2835.9 \pm 879.6 \mathrm{MPa}(\mathrm{p}=0.0662)$ respectively, representing an approximate reduction of $19 \%$ in the YM of the experimental group. Due to a large dispersion in the control group data, this result did not meet the $p \leq 0.05$ criteria associated with significance $[23,24]$. Table 1 presents the results of the seven parameters measured for both the control and experimental groups, with the relevant sample numbers and confidence levels. In Fig. 1, histograms (including errors) summarizing the complete quantitative data set in relative terms, are presented. The bars are normalized such that the control mean value is set to unity (with the exception of Fe concentration, where the experimental mean is set to unity instead, to avoid geometric scaling complications).

Table 1 Results of the seven parameters measured, for both the control and experimental groups, with the relevant sample numbers and confidence levels

\begin{tabular}{llll}
\hline & Control & Experimental & p (one-tail) \\
\hline TH $(\mathrm{mm})$ & $0.61 \pm 0.07(\mathrm{n}=6)$ & $0.48 \pm 0.03(\mathrm{n}=6)$ & 0.0500 \\
$\mathrm{CD}\left(\mathrm{g} / \mathrm{cm}^{3}\right)$ & $1.9785 \pm 0.1948(\mathrm{n}=9)$ & $1.7698 \pm 0.2223(\mathrm{n}=9)$ & 0.0251 \\
$\mathrm{BMD}\left(\mathrm{g} / \mathrm{cm}^{2}\right)$ & $0.152 \pm 0.005(\mathrm{n}=6)$ & $0.143 \pm 0.005(\mathrm{n}=6)$ & 0.0089 \\
$\mathrm{Ca}(\mathrm{ppm})$ & $82621.8 \pm 11046.7(\mathrm{n}=9)$ & $70912.8 \pm 11952.7(\mathrm{n}=9)$ & 0.0272 \\
Fe $(\mathrm{ppm})$ & $80.87 \pm 37.41(\mathrm{n}=9)$ & $242.76 \pm 190.35(\mathrm{n}=9)$ & 0.0127 \\
BS $(\mathrm{N})$ & $451.57 \pm 133.78(\mathrm{n}=7)$ & $328.50 \pm 139.62(\mathrm{n}=8)$ & 0.0500 \\
YM $(\mathrm{MPa})$ & $3483.6 \pm 1853.7(\mathrm{n}=7)$ & $2835.9 \pm 879.6(\mathrm{n}=8)$ & 0.0662 \\
\hline
\end{tabular}

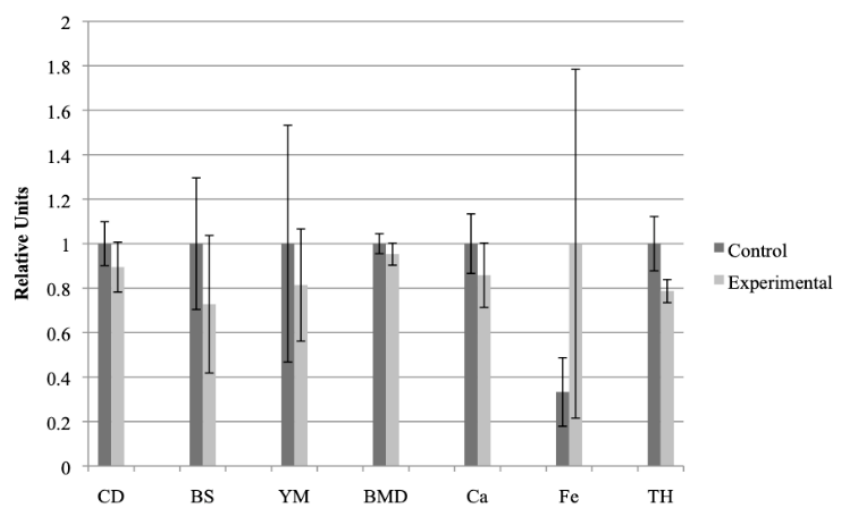

Fig. 1 Histograms summarizing the complete quantitative data set in relative terms. The bars are normalized such that the control mean value is set to unity (with the exception of Fe concentration)

Electron micrographs of cortical tibio-tarsi of a representative sample from the control group (normal diet) on left, and experimental group (slag diet) on right are presented in Figs. $2 \mathrm{a}$ and $\mathrm{b}$ respectively. Loss of granular architecture in the experimental sample is consistent with bone resorption. In particular, the typically larger cavities observed are consistent with resorptive cavities lined with osteoclasts. The observed https://doi.org/10.30799/jespr.171.19050205 changes may also be indicative of cortical discontinuities, foci of woven bone formation, and/or spicules of fibroplasia, consistent with previously reported histological findings [1]. Brissot et al. described generally similar changes in bony structures in humans subjected to iron overload conditions [25]. Although not directly relevant to this work, for purposes of completeness we mention the work of Conrad et al. who addressed soft tissue changes induced by iron overload in humans [16]. Follow up EDS analysis (areas inside the rectangles) revealed the presence of elemental sulfur at a concentration of $2.61 \pm 0.02 \%$ by mass fraction in the experimental sample, with no sulfur detected in the control sample. This correlates well with the reported $2.5 \pm 0.5 \%$ sulfur concentration by mass in nickel recovery slag in the Sudbury basin [2]. No reports were found in the literature regarding sulphur uptake in bones in animals of any type fed a slag-rich diet, potentially making this the first such report.
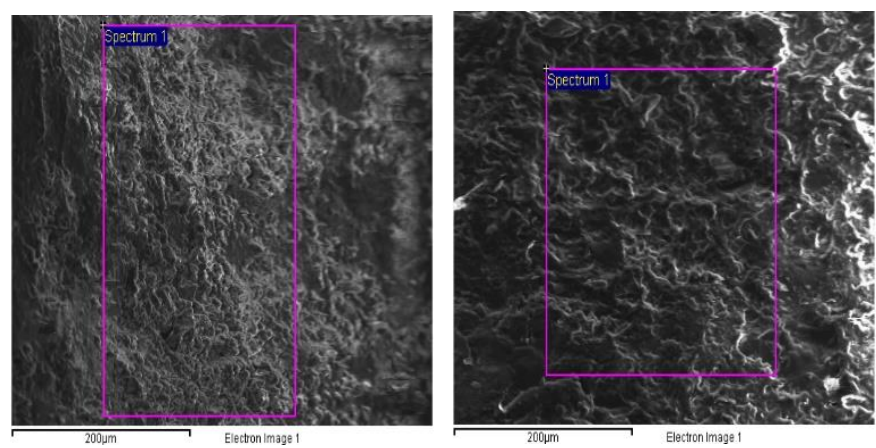

Fig. 2 Electron micrographs of a) cortical tibio-tarsus of a representative sample from the control group (limestone grit) on left, and b) experimental group (slag grit) on right. Note structural changes in the experimental sample, consistent with resorption, woven formations, and fibroplasia

Figs. 3a and b are section optical micrographs of cortical tibio-tarsi of the control group sample (normal diet) on left, and experimental group sample (metal diet) on right. The micrographs reveal a thinning of the cortical thickness of the experimental sample, from approximately $600 \mu \mathrm{m}$ to $500 \mu \mathrm{m}$, for an approximate $15 \%$ reduction in thickness. The periosteal and endosteal borders appear irregular and discontinuous in the experimental sample, with protuberances in the periosteum and indentations in the endosteum, consistent with resorption. None of these abnormalities are apparent in the control sample, which presents smooth periosteal and endosteal surfaces.

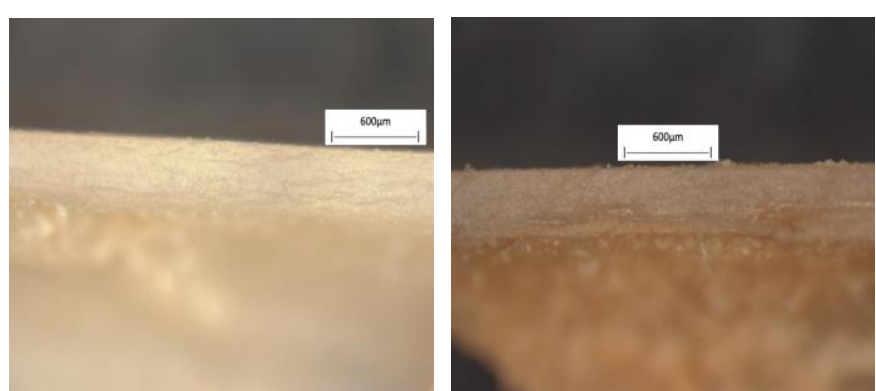

Fig. 3 Optical micrographs of a) cortical tibio-tarsi of control group sample (limestone grit) on left, and b) experimental group sample (slag grit) on right. Note the thinning of the cortical thickness, as well as the increased irregularity of the periosteal and endosteal surfaces in the experimental sample

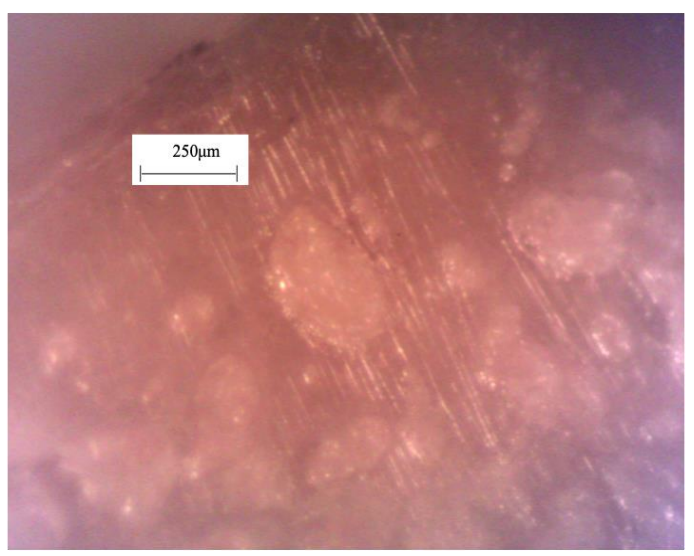

Fig. 4 The experimental sample revealed the presence of ovoid shaped, granular cavities surrounded by parallel-arranged spicules. No such structures were observed in the control sample 
Further conventional microscopy analysis of the experimental sample revealed the presence of ovoid shaped granular cavities, ranging in diameter between $50 \mu \mathrm{m}$ and $200 \mu \mathrm{m}$. These cavities were surrounded by parallel-arranged spicules, as presented in Fig. 4. The cavities contain increased quantities of osteoclasts, in effect constituting resorptive cavities, compatible with electron microscopy findings. The spicular structures appear to be areas with overrepresentation of osteoblasts and/or fibroplastic changes, however further investigation is needed to confirm this. No such structures were observed in the control sample.

Optical microscopy at the highest available magnification (1500x), revealed the existence of a red-brown localized circular deposit - of approximate diameter of $40 \mu \mathrm{m}$ - on the cortical structure of the experimental sample, as seen in Fig. 5. On follow-up EDS analysis of the specific spot, an approximate eightfold increase in the Fe concentration (by mass fraction) was found relative to the surrounding cortical bone. The mean Fe mass fraction within the area of the spot was $0.22 \pm 0.01 \%$, compared to a mean value $0.026 \pm 0.001 \%$ for the surrounding bone. Considering that our mass spectrometry measurements have shown a three-fold increase in (mean) Fe concentration in the experimental samples, this additional EDS result implies that the localized deposit exhibits a 24-fold increase in Fe concentration compared to the mean values for the control group. Based on abnormally high Fe concentrations reported in the liver and kidneys of these very same laboratory animals, a condition of hemosiderosis had been proposed [1]. Previous reports of avian hemosiderosis exist, but none specifically addressed Fe concentrations in bones $[11,12]$. This is believed to be the first report of a localized deposit of abnormally high Fe concentration in bone in an avian sample with known hemosiderosis. No such deposits were observed in our control sample, despite determined efforts to identify them.

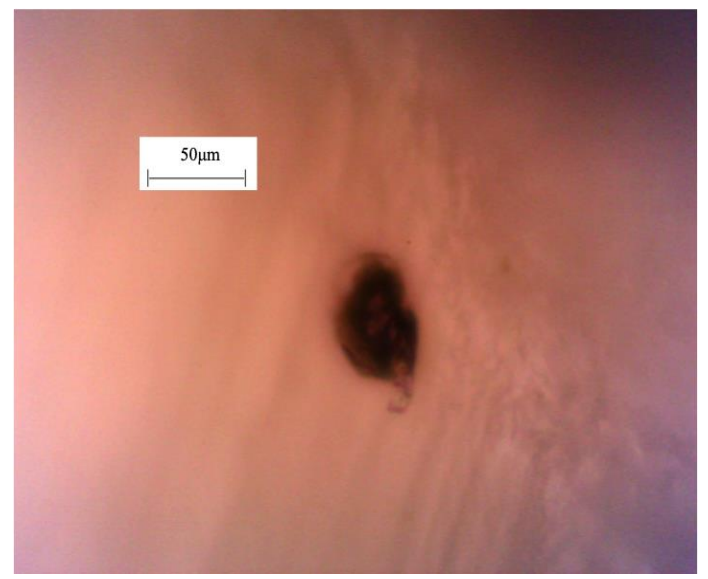

Fig. 5 Optical micrograph of a red-brown localized deposit, of approximate diameter of $40 \mu \mathrm{m}$, on the cortical structure of the experimental sample. EDS analysis of the spot revealed an abnormally high concentration of Fe

The last part of our investigation involved hardness measurements of dietary components of the two groups. Brinell testing yielded a mean number of $81.2(n=3)$ for slag and $35.4(n=4)$ for limestone, while Rockwell testing yielded numbers of 634 and 327 (both $n=1$ ), for slag and limestone respectively. An approximate hardness ratio of $2: 1$ for slag vs. limestone supported by both independent testing methods, yields a robust conclusion in this case. The greater mechanical hardness of slag very likely played a role in its well documented slower rate of in-vivo erosion, and greater retention time in the gastric system [1].

\section{Conclusion}

In six of the seven quantitative functional bone parameters tested, statistically significant degradations were measured. A seventh parameter (Young's Modulus) also recorded a degradation, but slightly outside of the chosen statistical significance parameter ( $p \leq 0.05)$. Electron microscopy revealed resorptive activity in the tibio-tarsi of the metal diet group, while optical microscopy revealed the presence of cortical irregularities and confirmed the presence of resorptive foci. The presence of a single reddish-brown spot in the experimental group sample detected under optical microscopy, revealed a localized high-concentration Fe deposit under follow-up electron dispersive spectroscopy (EDS). EDS also revealed the uniform presence of elemental sulphur in the experimental group sample, consistent with known sulphur levels in dietary slag; no sulphur was detected in the control sample examined. Engineering testing revealed that slag-based dietary components of the experimental group were approximately twice as hard (Brinnell and Rockwell hardness) as the dietary components of the control group. In summary, based on the measurements performed in this work, we conclude that the dietary intake of nickel recovery slag is associated with measurable mechanical, chemical, and radiological changes - as well as detectable qualitative structural changes - in the tibio-tarsi of Columbia Livia Domestica pigeons.

\section{References}

[1] G.H. Parker, G. Van Der Kraak, Use of metal-rich slag as a source of grit and its effects on pigeon health and fitness, J. Env. Sci. Pollut. Res. 4(2) (2018) pp 256267.

[2] NSL, The leaching of slag from blast furnace, National Slag Limited, Canada 1985.

[3] W.K. Kim, L.M. Donalson, P. Herrera, C.L. Woodward, L.F. Kubena, et al., Effects of different bone preparation methods (fresh, dry, and fat-free dry) on bone parameters and the correlations between bone breaking strength and the other bone parameters, Poult. Sci. 83 (2004) 1663-1666.

[4] A. Pietrobelli, C. Formica, Z. Wang, S.B. Heymsfield, Dual-energy X-ray absorptiometry body composition model: review of physical concepts, Am. J. Physiol. Endocrinol. Metabol. 271(6) (1996) 941-951.

[5] Zinke-Allmang, R. Nejat, E. Galiano-Riveros, J. Bayer, M.X. Chen, Elasticity and vibrations, In: Physics for the Life Sciences. $3^{\text {rd }}$ Edn., Nelson Education Ltd. Canada, 2017, pp.346-351.

[6] M. Philip, A. Nahum, J.C. Rosenwald, Handbook of Radiotherapy Physics: Theory and Practice, CRC Press, Boca Raton, FL, USA, 2007, pp.36-39.

[7] E.M. Tobolski, A. Fee, Macroindentation hardness testing, In: ASM Handbook, Vol. 8, Mechanical testing and evaluation, ASM International, Ohio, 2000, pp.203-211.

[8] ASTM E10-14, Standard test method for Brinell hardness testing of materials, ASTM InternationalWest Conshohocken, PA, 2014

[9] J. Tsay, Z. Yang, F.P. Ross, S. Cunningham-Rundles, H. Lin, et al., Bone loss due to iron overload in a murine model: importance of oxidative stress, Blood 116 (2010) 2582-2589.

[10] M.C. De Vernejoul, A. Pointillart, C.C. Golenzer, C. Morieux, J. Bielakoff, et al., Effects of iron overload on bone remodeling in pigs, Am. J. Pathology 116(3) (1984) 377-382.

[11] S.C. Cork, M.R. Alley, P.H.G. Stockdale, A quantitative assessment of hemosiderosis in wild and captive birds using image analysis, Avian. Pathol. 24 (1995) 239-254

[12] S.C. Cork, Iron storage disease in birds, Avian. Pathol. 29 (2000) 7-12.

[13] T.K. Cheng, C.N. Coon, Effect of calcium source, particle size, limestone solubility in vitro, and calcium intake level on layer bone status and performance, Poult. Sci. 69(12) (1990) 2214-2219.

[14] J.I. Orban, Factors influencing bone mineral content, density, breaking strength and ash as response criteria for assessing bone quality in chickens, Poult. Sci. 72(3) (1993) 437-446.

[15] R.J. Ward, T.C. Iancu, G.M. Henderson, J.R. Kirkwood, T.J. Peters, Hepatic iron overload in birds: analytical and morphological studies, Avian Pathol. 17 (1988) 451-464

[16] Conrad, E. Marcel, Jay N. Umbreit, Iron absorption and transport - an update. Am. J. Hematol. 64(4) (2000) 287-298.

[17] P.Y. Hester, M.A. Schreiweis, J.I. Orban, H. Mazzuco, M.N. Kopka, et al., Assessing bone mineral density in vivo: dual energy X-ray absorptiometry, Poult. Sci. 83(2) (2004) 215-221.

[18] X.G. Cheng, G. Lowet, S. Boonen, P.H. Nicholson, P. Brys, et al., Assessment of the strength of proximal femur in vitro: relationship to femoral bone mineral density and femoral geometry, Bone 20(3) (1997) 213-218.

[19] B.J. Kim, S.H. Lee, J.M. Koh, G.S. Kim, The association between higher serum ferritin level and lower bone mineral density is prominent in women $\geq 45$ years of age, Osteo. Int. 24(10) (2013) 2627-2637.

[20] G.M. Dorrestein, G.M. Grinwis, L. Dominguez, E. Van de Jagt, A.C. Beynen, An induced iron-storage disease syndrome in doves and pigeons: a model for haemochromatosis in mynah birds? In: Proceedings of the Association of Avian Veterinarians, New Orleans, LA, 1992, pp.108-112.

[21] G. Huyghebaert, Effect of dietary fluoride on performances and bone characteristics of broilers and the influence of drying and defatting on bonebreaking strength, Poult. Sci. 67(6) (1988) 950-955.

[22] B. Zhang, C.N. Coon, The relationship of various tibia bone measurements in hens, Poult. Sci.76(12) (1997) 1698-1701.

[23] M.L. Samuels, J.A. Witmer, A. Schaffner, The t-test in Statistics for the Life Sciences, $5^{\text {th }}$ Edn., Pearson Inc., USA, 2016.

[24] P. Bevington, D.K. Robinson, Confidence intervals in data reduction and error analysis for the physical sciences, 3rd Edn., McGraw-Hill, USA, 2002, pp.208210.

[25] Brissot, Pierre, M. Ropert, C. Le Lan, O. Loréal, Non-transferrin bound iron: a key role in iron overload and iron toxicity, Biochim. Biophys. Acta (BBA)-Gen. Sub. 1820(3) (2012) 403-410. 